\title{
Vector magnetic field map at the photospheric level below and around a solar filament (neutral line) ${ }^{\star}$
}

\author{
V. Bommier ${ }^{1}$, J. Rayrole ${ }^{2}$, and A. Eff-Darwich ${ }^{3}$ \\ ${ }^{1}$ Laboratoire d'Étude du Rayonnement et de la Matière en Astrophysique, CNRS UMR 8112 - LERMA, Observatoire de Paris, \\ Section de Meudon, 92195 Meudon, France \\ e-mail: v. bommier@obspm. fr \\ 2 Laboratoire d'Études Spatiales et d'Instrumentation en Astrophysique, CNRS UMR 8109 - LESIA, Observatoire de Paris, \\ Section de Meudon, 92195 Meudon, France \\ 3 THEMIS S.L., via Lactea s/n, 38200 La Laguna, Tenerife, Islas Canarias, Spain
}

Received 30 August 2004 / Accepted 17 January 2005

\begin{abstract}
We present a vector magnetic field map obtained on 7 December 2003, below and around a filament located not so far from the active region NOAA 517, whose one spot is also found on the map of $240 \times 340$ arcsec. This region was itself located near the disk center, so that the longitudinal (resp. transverse) field is nearly the vertical (resp. horizontal) one. The THEMIS telescope was used in its spectropolarimetric multiline mode MTR ("MulTiRaies"). The noise level is 5-10 Gauss in the longitudinal field and 50-100 Gauss in the transverse field, while the pixel size is 0.45 arcsec. Fundamental ambiguity is not solved, and the atmosphere is assumed to be homogeneous. The magnetic field derivation method described in this paper was validated on eight test points submitted to the UNNOFIT inversion code, and the results are found in agreement within 14\% discrepancy. Two main results appear on the map: (i) a strong spatial correlation between the longitudinal and transverse field resulting in an inclined field vector (making a most probable angle of $60^{\circ}$ or $120^{\circ}$ with the line-of-sight in the filament region); and (ii) homogeneity of the field direction (inclination and azimuth) in the filament region. Parasitic polarities were also detected: first those located at the filament feet, as theoretically expected, on the one hand; and then weak opposite polarity regular patterns that appear between the network field (strong field at the frontiers of supergranules), on the other. The exact superimposition of the magnetic field map derived from the Fe I $6302.5 \AA$ line and of the $\mathrm{H} \alpha$ map, which enabled association of the parasitic polarities with the filament feet, was possible because these two maps were simultaneously obtained, thanks to a unique facility available in the multiline mode of THEMIS.
\end{abstract}

Key words. Sun: magnetic fields - polarization - Sun: filaments - Sun: prominences

\section{Introduction}

The aim of the present work was to provide a vectorial magnetic field map of the photospheric field below and around a filament, in order to fix some boundary conditions for extrapolation of the magnetic field in the filament, following for instance the method of Aulanier \& Démoulin $(1998,2003)$. To this purpose, the filament must be fully scanned in length, and in width the map dimension must be 3 times the filament length, in order to describe the neighbouring polarities fully. These conditions were fulfilled by our observation, because the filament is about 2 arcmin long, extending horizontally on the map, which is $240 \times 340$ arcsec (see Fig. 1).

* Based on observations made with the French-Italian telescope THEMIS operated by the CNRS and CNR on the island of Tenerife in the Spanish Observatorio del Teide of the Instituto de Astrofísica de Canarias.
The spectropolarimetric multiline mode MTR ("MulTiRaies") of the telescope THEMIS was used. The images are spectral in one dimension and spatial in the other, and a map is reconstructed from a scan. The polarimetric analysis is performed before any inclined reflection, thus avoiding major contributions to instrumental polarization. The polarization analyzer includes a beamsplitter, which was proposed for use in the beam exchange technique (hereafter BE technique, Donati et al. 1990). This is a powerful technique for increasing polarimetric accuracy, but on time and space averaged data only (Bommier \& Molodij 2002). In the present program of a solar region scan, the data are not averaged, so that the BE technique's limit is given by the fact that the two solar images taken with the two analyzer positions should show neither time evolution nor spatial displacement. Nevertheless, the BE correcting effect of the channel difference deserves interest and further investigation. 
After a brief description of the instrument, we will present our methods for: a) observations (Sect. 2); b) data reduction (Sect. 3); and c) magnetic inversion (Sect. 4). The results are presented in Sect. 5 and discussed in Sect. 6, where comparison is made with other instruments.

\subsection{Brief instrument description}

The instrument's particularity is to be "polarization free", as stated above. Polarization sensitivity is limited by the major source of noise, the photon noise. As the detectors have a potential well of $N=10^{6}$ electrons, the photon noise limited polarization sensitivity is at most $1 \sqrt{N}=10^{-3}$ and is usually $2 \times 10^{-3}$ for each pixel of each image. The instrument is "multiline", which means that several lines can be simultaneously recorded on different cameras. For these observations, we observed five different spectral windows, each in the two polarization states, resulting in 10 cameras plus one slitjaw. The spectral resolution was $24 \mathrm{~m} \AA$ at $6302 \AA$.

A more detailed description of the THEMIS instrument can be found in Arnaud et al. (1998), though it has to be updated as regards the tip-tilt correction, and the polarization analyzer quarter-wave plate positions that are now free to take any position needed.

\section{Observations}

The map was obtained in 3 scans of 120 arcsec width with a pixel size of 0.45 arcsec, leading to a spatial resolution of 0.9 arcsec (two times the pixel size). Accordingly, the scan was made of 300 steps of 0.8 arcsec each. The BE was performed in each of the polarization Stokes parameters $Q, U$, and $V$, leading to 6 successive analyzer positions for each slit position. The total observation duration was 9h08-14h06 UT. 5 different spectral windows were recorded: one on Fe I 6301.5 and $6302.5 \AA$; one on the Zeeman sensitive Fe I $5250.2 \AA$ and its 3 neighbouring lines; one on the unpolarizable Fe I 5576.1 $\AA$ for calibration; one on hydrogen $\mathrm{H} \alpha$, and one on the Ti I 5565.48 $\AA$ line that is sensitive to atomic polarization for solving the fundamental ambiguity, following the method proposed by Landi Degl'Innocenti \& Bommier (1993).

\section{Data reduction}

The line position method and FFT techniques described in Bommier \& Rayrole (2002) were used for line destretching, line alignment, and anamorphosis correction. Particular attention were devoted to cospatiality corrections parallel and perpendicular to the slit. The cospatiality parallel to the slit and between the two beams was corrected after having measured the misalignment by correlation on the velocity along the slit, determined after summing the $I+S$ and $I-S$ data provided in each beam by BE, where $S$ is a generic symbol for the Stokes parameter (avoiding thus the large magnetic perturbation due to the spot). By dividing the slit into two parts, it is possible to derive the magnifying difference, which can then also be corrected. The cospatiality perpendicular to the slit can be measured on the velocity maps of the full scan in each beam, obtained as described above from the BE data. The correlation is able to provide difference in solar position between both beams, which can be converted from the step size in mixing factor between successive images, though it has not been demonstrated that this method corrects the cospatiality difference. When both cospatiality corrections parallel and perpendicular to the slit are performed, it is necessary to do iterations from one to the next. The dispersion difference (also perpendicular to the slit) is easily derived from neighbouring line positions, so that the correction can be performed. Fortunately, the settings were found good enough (slit parallelism difference 0.065 arcsec, slit position mean difference 0.011 arcsec - the slit width being 0.5 arcsec -, magnifying factor difference $0.04 \%$, dispersion difference $0.32 \%$ ) so that only the correction of cospatiality parallel to the slit was really necessary.

\section{Magnetic field derivation}

The following methods were used for a direct though approximate determination of the field. Results were compared to those given by a more sophisticated inversion technique on eight test points labelled from $\mathrm{A}$ to $\mathrm{H}$ in Fig. 1.

\subsection{Longitudinal magnetic field}

The longitudinal magnetic field was determined from the difference between line positions determined by using the bisector method in $I+V$ and $I-V$ images, denoted as $\Delta \lambda_{+}-\Delta \lambda_{-}$. From the Zeeman effect law, associated to geometrical considerations, it can be shown (Semel 1967) that

$\Delta \lambda_{+}-\Delta \lambda_{-}=4.67 \bar{g} \lambda_{0}^{2} \frac{B \cos \theta}{50}$

where $\Delta \lambda$ is in $\mathrm{m} \AA$ and the central wavelength $\lambda_{0}$ is in $\mu \mathrm{m}$; $B$ is the field strength in Gauss and $\theta$ the angle between the magnetic field vector and the line-of-sight. $\bar{g}$ is the effective Landé factor given by

$\bar{g}=\frac{g_{\mathrm{s}}}{2}+J_{\mathrm{d}} \frac{g_{\mathrm{d}}}{4}$

where

$g_{\mathrm{s}}=g_{u}+g_{\ell}$

$g_{\mathrm{d}}=g_{u}-g_{\ell}$

and

$J_{\mathrm{s}}=\left[J_{u}\left(J_{u}+1\right)+J_{\ell}\left(J_{\ell}+1\right)\right]$

$J_{\mathrm{d}}=\left[J_{u}\left(J_{u}+1\right)-J_{\ell}\left(J_{\ell}+1\right)\right]$

where $u$ and $\ell$ denote the upper and lower levels respectively, and $g$ is the Landé factor of a level of total kinetic momentum $J . \bar{g}=2.5$ for Fe I $6302.5 \AA$.

No weak field approximation was done, so that the law remains valid even for strong fields. Nevertheless, the transfer effects were ignored here, which can lead to inaccuracy of about $10 \%$ in certain strong field cases, as shown by Semel (1967). The noise level in the longitudinal field determination has been found to be 5-10 Gauss. 


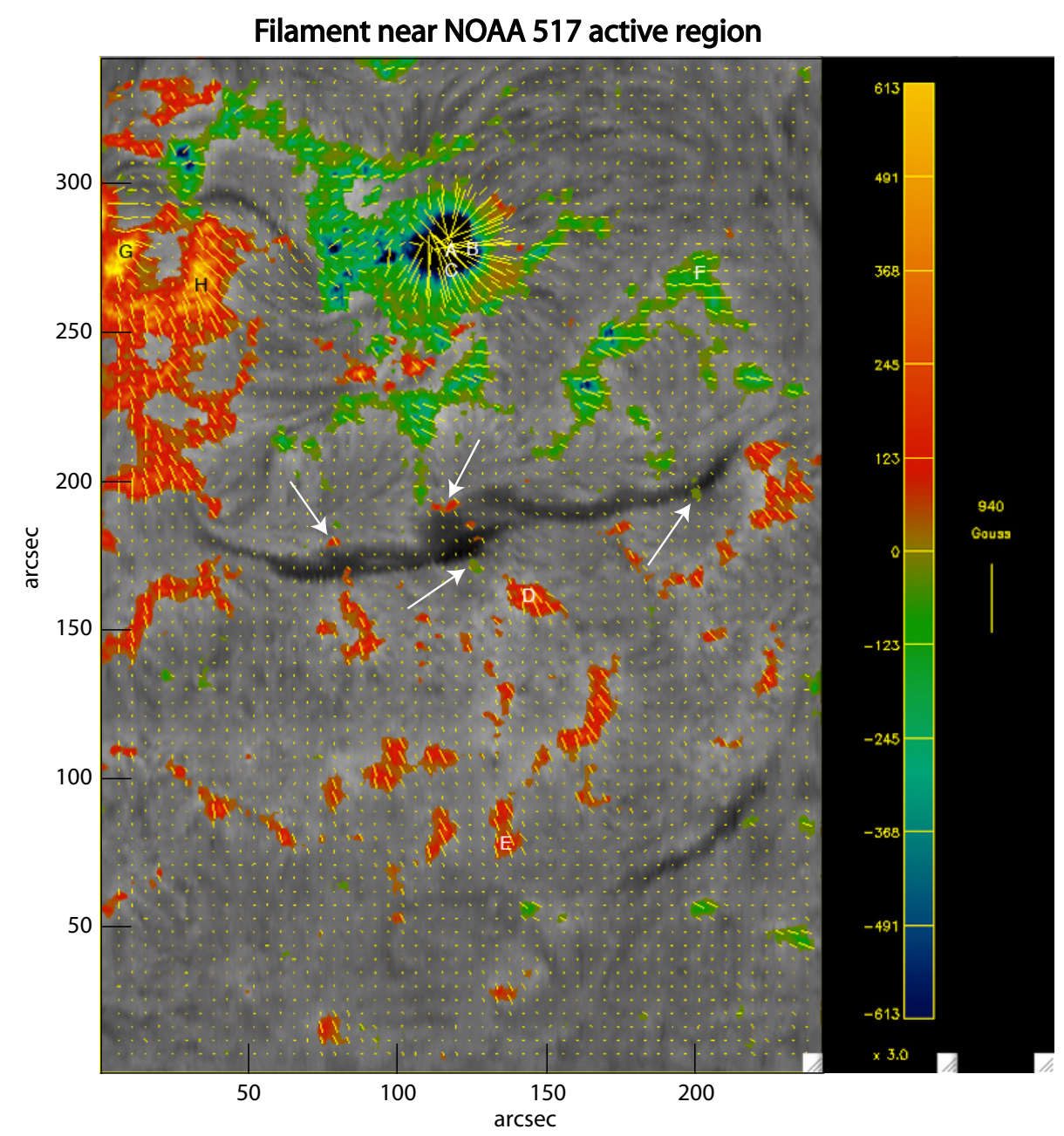

Fig. 1. The map obtained on 7 December 2003 in Fe I $6302.5 \AA$ and composed of 3 superimposed layers. The bottom layer is the H $\alpha$ image; the colored layer displays the longitudinal field, saturated inside the spot, where the maximum field is $3 \times 613$ Gauss; the dash layer displays the transverse field, unresolved in orientation due to the fundamental ambiguity. The white arrows point to parasitic polarities at the filament feet. The letters from A to $\mathrm{H}$ localize the 8 points where the UNNOFIT inversion code has been applied to test our magnetic field diagnostic method.

\subsection{Transverse magnetic field}

The tranverse field was derived from the weak field law (Landi Degl'Innocenti 1992a)

$$
\begin{aligned}
B \sin \theta= & 1.71 \times 10^{5} \frac{1}{\sqrt{\bar{g}^{2}+\epsilon_{2}}}\left(\frac{\lambda_{0}}{5000}\right) \\
& \times \sqrt{\frac{p_{\ell}\left(\lambda_{0}\right) I\left(\lambda_{0}\right)}{\left[\mathrm{d}^{2} I / \mathrm{d} \lambda^{2}\right]_{\lambda=\lambda_{0}}}},
\end{aligned}
$$

where the central wavelength $\lambda_{0}$ is now in $\AA$. The second derivative of the intensity $I$ profile is taken at line center, and $p_{\ell}$ is the linear polarization degree

$p_{\ell}=\sqrt{(Q / I)^{2}+(U / I)^{2}}$

taken at line center, and $\epsilon_{2}$ is a coefficient given by

$\epsilon_{2}=g_{\mathrm{d}}^{2} \frac{4-16 J_{\mathrm{s}}+7 J_{\mathrm{d}}^{2}}{80}$,

while $\epsilon_{2}=0$ for Fe I $6302.5 \AA$, the magnetic field strength is given in Gauss. The second derivative of the $I$ profile was in fact determined in the Fe I 5576.1 scan that is insensitive to the magnetic field, in order to avoid spot perturbation, and then was rescaled to Fe I 6302.5 by using the flat-field data. Due to this "calibration", the method's accuracy, which is theoretically limited to a weak field, is extended to a higher field as shown by comparing inversion code results (see the next subsection).

The transverse field direction is given modulo $\pi$ by

$\alpha=\frac{1}{2} \frac{-U\left(\lambda_{0}\right)}{\left|U\left(\lambda_{0}\right)\right|} \arccos \left[\frac{-Q\left(\lambda_{0}\right)}{\sqrt{Q^{2}\left(\lambda_{0}\right)+U^{2}\left(\lambda_{0}\right)}}\right]$.

The noise level in the transverse field determination was found to be 50-100 Gauss.

\subsection{Validation of the method}

The method has been validated by comparison of the results given in eight test points with those provided by the UNNOFIT inversion code (Landolfi et al. 1984). These test points are labelled from $\mathrm{A}$ to $\mathrm{H}$ in Fig. 1 and are located in stronger and weaker field regions, in the spot umbra, 
Table 1. Test of our magnetic field diagnostic method with the inversion code UNNOFIT. The points from A to $\mathrm{H}$ are localized on the map in Fig. 1, while those following compare results of the diagnostic method described in the present paper (Index 1) with results of the UNNOFIT inversion code (Index 2), for the longitudinal magnetic field $B_{/ /}$, the transverse magnetic field strength $B_{\perp}$, the transverse field azimuth direction referred with respect to the slit direction, which is also the meridian (map $O y$ axis), the strength of the field vector $B_{\text {tot }}$, and the inclination angle with respect to the line-of-sight.

\begin{tabular}{crrrrrrrrrr}
\hline \hline Point & $\begin{array}{r}B_{/ /} \\
\text {Gauss }\end{array}$ & $\begin{array}{r}B_{/ /} \\
\text {Gauss }\end{array}$ & $\begin{array}{r}B_{\perp} \\
\text { Gauss }\end{array}$ & $\begin{array}{r}B_{\perp} \\
\text { Gauss }\end{array}$ & $\begin{array}{r}\text { Azimuth } \\
\text { degree }\end{array}$ & $\begin{array}{r}\text { Azimuth } \\
\text { degree }\end{array}$ & $\begin{array}{r}B_{\text {tot }} \\
\text { Gauss }\end{array}$ & $\begin{array}{r}B_{\text {tot }} \\
\text { Gauss }\end{array}$ & $\begin{array}{r}\text { Inclination } \\
\text { degree }\end{array}$ & $\begin{array}{r}\text { Inclination } \\
\text { degree }\end{array}$ \\
& 1 & 2 & 1 & 2 & 1 & 2 & 1 & 2 & 1 & 2 \\
\hline A & -1711 & -1958 & 365 & 479 & -86 & -126 & 1750 & 2016 & 168 & 166 \\
B & -787 & -849 & 1012 & 1403 & -82 & -90 & 1282 & 1640 & 128 & 122 \\
C & -1000 & -1054 & 1000 & 1267 & +23 & +10 & 1414 & 1648 & 135 & 130 \\
D & +269 & +341 & 222 & 207 & +21 & +35 & 349 & 341 & 40 & 37 \\
E & +103 & +99 & 170 & 198 & +20 & +35 & 199 & 221 & 59 & 63 \\
F & -93 & -87 & 200 & 153 & -89 & -108 & 221 & 176 & 115 & 120 \\
G & +952 & +828 & 436 & 548 & +21 & +35 & 1047 & 993 & 25 & 34 \\
H & +570 & +556 & 452 & 485 & +38 & +48 & 728 & 738 & 38 & 41 \\
\hline
\end{tabular}

the penumbra and in the filament region. As the larger discrepancies are expected in the strong field regions, several points are located in such regions. The UNNOFIT inversion code is based on the Marquardt algorithm to reach the minimum theory/observation discrepancy with the theoretical profiles given by the Unno-Rachkowski solution. Pionereed by Harvey et al. (1972) and Auer et al. (1977), this technique has been improved by Landolfi and by Landi Degl'Innocenti (1982) and Landolfi et al. (1984) to allow for magneto-optical and damping effects. In the present analysis, the atmosphere was assumed to be homogeneous, and no filling factor was introduced for the magnetic field.

The results of the comparison are given in Table 1 for the various field coordinates. As a mean evaluation, our method agrees with the results of the inversion code within $14 \%$ inaccuracy, even in the strong field regions.

\section{Results}

The vectorial magnetic field map is given in Fig. 1, where two main features appear: a) the strong correlation between vertical and horizontal field; b) the homogeneity of the field direction in the filament zone. These results are discussed in more detail below. We also investigated the weak field regions by plotting the "logarithmic map"

$\frac{B_{/ /}}{\left|B_{/ /}\right|} \log _{10}\left(1+\left|B_{/ /}\right|\right)$,

the longitudinal field strength being taken in Gauss. This map is given in Fig. 2.

\subsection{Parasitic polarities}

The vectorial map of Fig. 1 displays the filament lying in image center, and one of the two spots of the NOAA 517 active region in the image upper part (half of the second spot is visible on the left edge). Colors designate the longitudinal magnetic field: cold colors (blue, green) for the (negative) field entering the Sun, and hot colors (red, yellow) for the (positive) field coming out of the Sun. Yellow dashes $(10 \times 10$ pixels averaged for clarity) have length proportional to the transverse field strength, and direction that of the transverse field. Orientation of this field along its direction is unknown due to the fundamental ambiguity. NOAA 517 on 7 December 2003 was located near the disk center, so that the longitudinal (resp. transverse) field is nearly the vertical (resp. horizontal) one. The filament lies along the neutral line that separates the negative polarities (blue, green) from the positive ones (red, yellow). White arrows point to the parasitic polarities (red on the green side or green on the red side) that are located at the filament feet (appearing like thorns on the disk). These parasitic polarities are also designated by black arrows in Fig. 2, where the filament has been drawn with a thin red contour.

A recent magnetohydrodynamical model (Aulanier \& Démoulin 1998) shows that the parasitic polarities are essential for explaining formation of the magnetic structure that supports the filament, and equally fundamental in filament formation and stability.

\subsection{Weak field structures}

Regular patterns of weak opposite polarity also appear inside the network field (strong field at the frontiers of supergranules, which form a network of 30 arcsec or more mesh), and are also visible with the same spatial pattern in Fe I 6301.5, thus suggesting a solar origin for these patterns. Some of them are indicated by white arrows in Fig. 2 .

\subsection{Magnetic field inclination}

A strong correlation has been found between longitudinal and transverse field spatial structures. Each longitudinal field spatial structure, even if it is small, corresponds to a transverse 


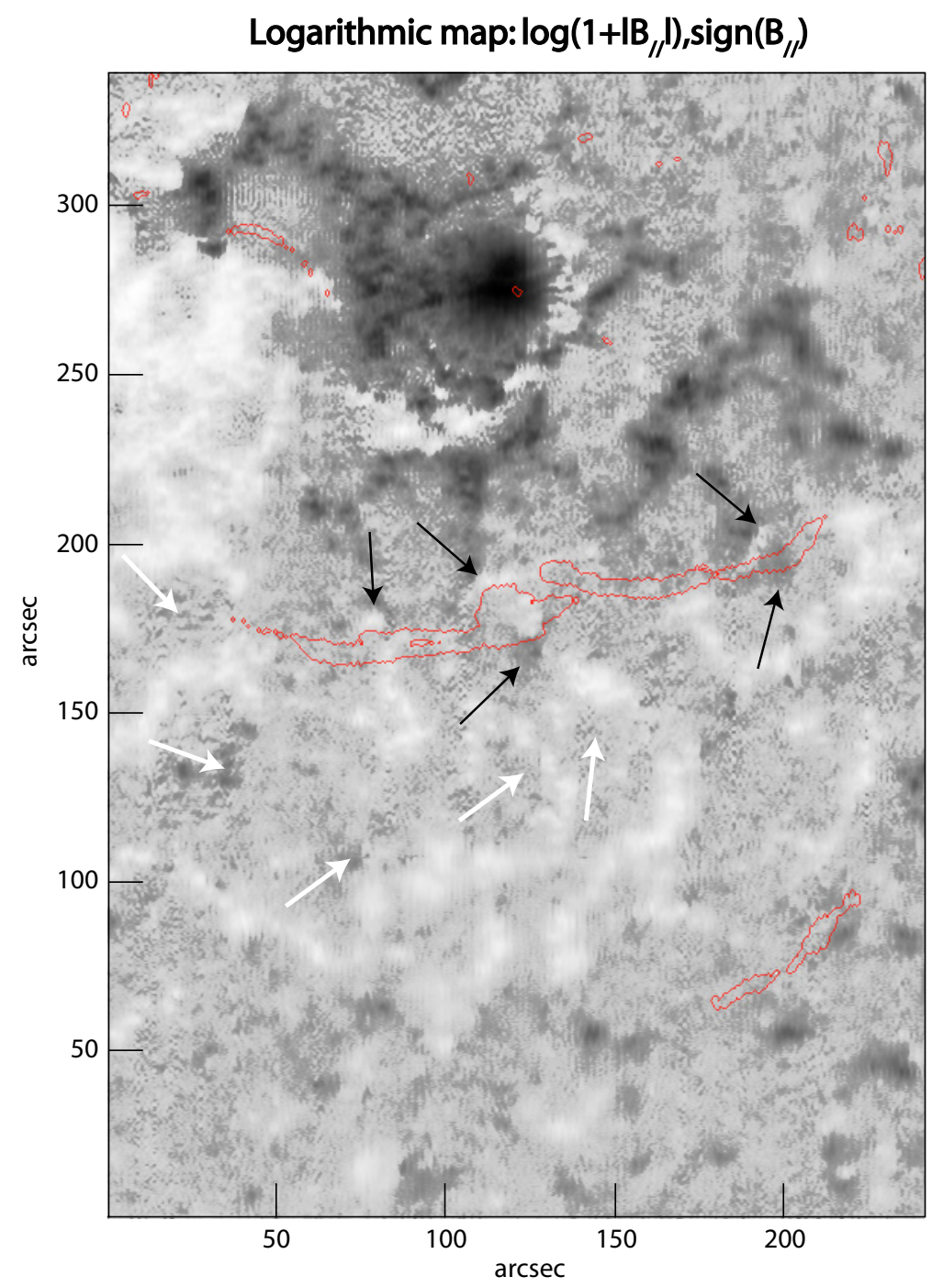

Fig. 2. Logarithmic map of the same region as in Fig. 1, i.e. the plotted field strength is $\log \left(1+\left|B_{/ /}\right|\right)$, to which the sign of the longitudinal field $B_{/ /}$is given (white for positive, black for negative). The black arrows point to the parasitic polarities at the filament feet and white arrows to some of the weak opposite polarity regular patterns that are also visible in Fe I $6301.5 \AA$. field one with the same shape, and vice versa. This can ben seen by a close comparison of the upper and lower parts of Fig. 3, where both longitudinal and tranverse field maps are displayed, one above the other (the particular behavior of the spot has been discarded). Correlation between vertical and horizontal fields is further investigated in Fig. 4, where for each pixel both lontigudinal and transverse field values have been plotted. Discarding those points that are below the noise level, which is 5-10 Gauss for the longitudinal field and 50-100 Gauss for the transverse field, it can be seen that the scatter of points does not cut the axes, thus demonstrating the correlation. This leads to a non-vertical vectorial field (except in the spot). Study of the inclination gives $60^{\circ}$ or $120^{\circ}$ as most probable angles between the field vector and the line-of-sight, as can be seen on the inclination histogram displayed in Fig. 5. This inclination histogram shows in addition that the inclination angle is quite homogeneous, except in the sunspot zone.

\subsection{Magnetic field azimuth}

The histogram of the magnetic field azimuth in the filament zone is displayed in Fig. 6, which shows that in this region the magnetic field azimuth is also quite homogeneous. Let us recall that because the fundamental ambiguity is not solved, the field azimuth is known modulo $\pi$ only.

\section{Discussion}

\subsection{Compared accuracy of the BE and non-BE techniques}

Investigations were made of the accuracy of the BE technique with respect to the quicker standard technique without BE. No difference in accuracy has been found for the longitudinal field determination from line positions. As for the transverse field that requires determination of $Q$ and $U$, the quicker "generalized BE" technique (exchange of one single Stokes parameter) proposed by Bommier \& Molodij (2002, BM02), has led to the same result as in BM02 (see their Table 4), namely an increase by a factor of 2 of the polarimetric noise in $Q$ and $U$ (factor of $\sqrt{2}$ for the transverse field), with BE performed in $V$. The "generalized BE" technique is nothing but a "demodulation" technique proposed by other authors (see for instance Trujillo Bueno et al. 2001). An additional increase by a factor of 2 in the polarimetric noise is found for the standard technique without any $\mathrm{BE}$. 


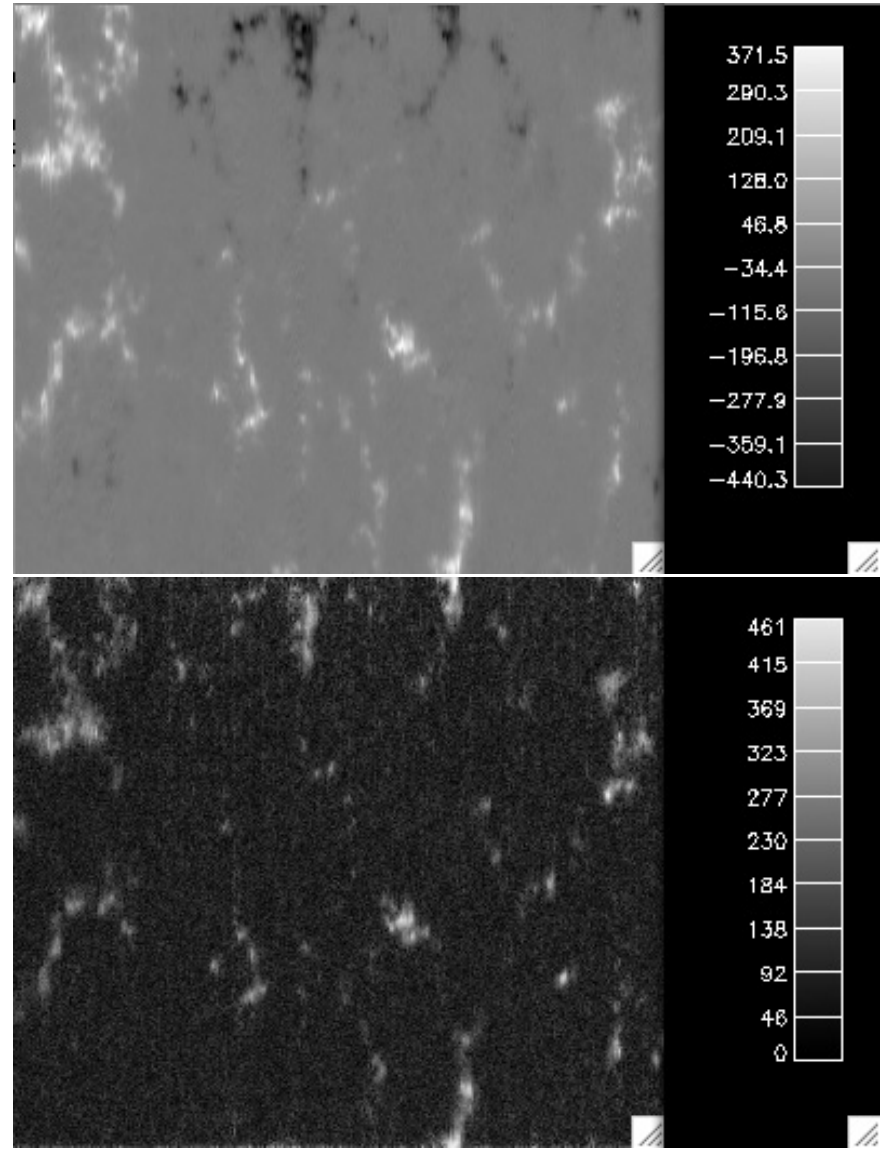

Fig. 3. For detailed comparison, longitudinal magnetic field (top) and transverse magnetic field intensity (bottom) of the central third of the map (not corrected for anamorphosis). Each detail of the longitudinal field map corresponds to a detail with same shape on the transverse field map.

\subsection{Comparison with other instruments}

Characteristics of the IVM ("Imaging Vector Magnetograph") at Haleakala, as described by Mickey et al. (1996), are equal or below those of THEMIS. Both instruments have the same polarimetric resolution $\left(10^{-3}\right)$. They have approximately the same pixel size ( 0.55 arcsec for IVM); but on IVM, pixels are binned $2 \times 2$ according to the seeing conditions to increase temporal resolution. The IVM field of view is $280 \times 280 \mathrm{arcsec}$, so comparable to the size of the present map. The main difference lies in the spectral resolution (70 mA on IVM, 3 times the one of THEMIS), which is probably the origin of higher calibration problems with IVM data.

The MSFC vector magnetograph (Hagyard et al. 1972) makes use of a $125 \mathrm{~m} \AA$ bandpass filter, leading to even greater calibration problems. The MSFC vector magnetograph has two modes: $5 \times 5$ arcmin field-of-view with 3.3 arcsec resolution, and $2 \times 2$ arcmin field-of-view with 1.3 arcsec resolution, a little below IVM performances.

The SOLIS instrument (Keller et al. 1998) is a full disk instrument with 1 arcsec spatial resolution. The polarimetric relative accuracy is also $10^{-3}$ (sensitivity $2 \times 10^{-4}$ ), and spectral resolution is $32 \mathrm{m \AA}$ at 6302 , comparable to the one of THEMIS. Temporal resolution is much better than THEMIS.

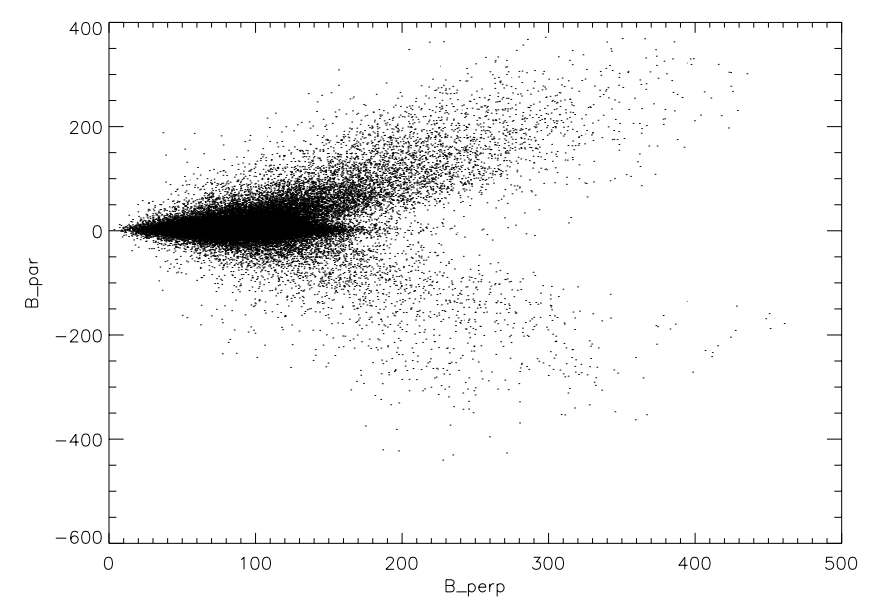

Fig. 4. Correlation between the transverse field $B_{\text {perp }}$ and the longitudinal field $B_{\mathrm{par}}$, for the central third of the map where the filament lies, thus avoiding the spot. No $B_{\text {par }}$ is found without a $B_{\text {perp }}$ contribution and vice versa, resulting in a non-vertical field. The noise level in the determination is 5-10 Gauss for $B_{\text {par }}$ and 50-100 Gauss for $B_{\text {perp. }}$.

B > 200 Gauss

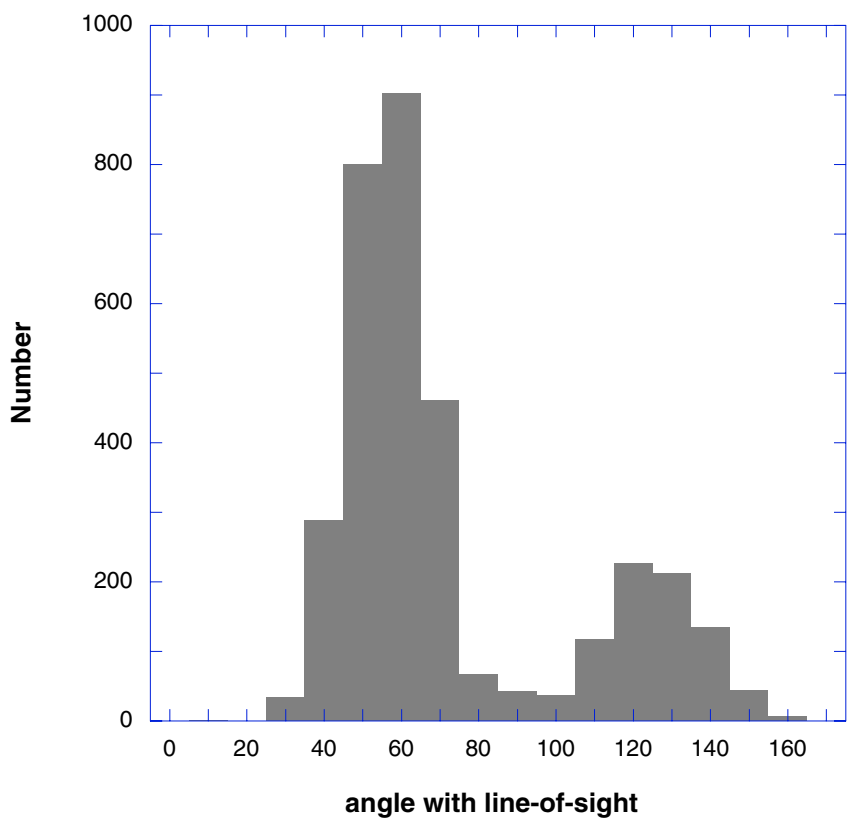

Fig. 5. Histogram of the the inclination angle with respect to line-ofsight in the central third of the map (the filament zone). Only the points where field strength $B_{\text {tot }}$ is larger than 200 Gauss were kept to build the histogram, which shows the homogeneity of the inclination direction in the filament zone.

However, no transverse magnetic field map obtained with this instrument has been made visible on the net for the moment.

The characteristics of the Advanced Stokes Polarimeter (ASP) implemented in the Dunn Solar Telescope of the High Altitude Observatory (HAO) are comparable to those of THEMIS. They are listed in Elmore et al. (2005): spatial resolution $<1$ arcsec for ASP, at least 0.8 arcsec (two times the pixel size) for THEMIS; polarimetric resolution better than $1 \times 10^{-3}$ for ASP (Skumanich et al. 1997), $2 \times 10^{-3}$ for THEMIS 


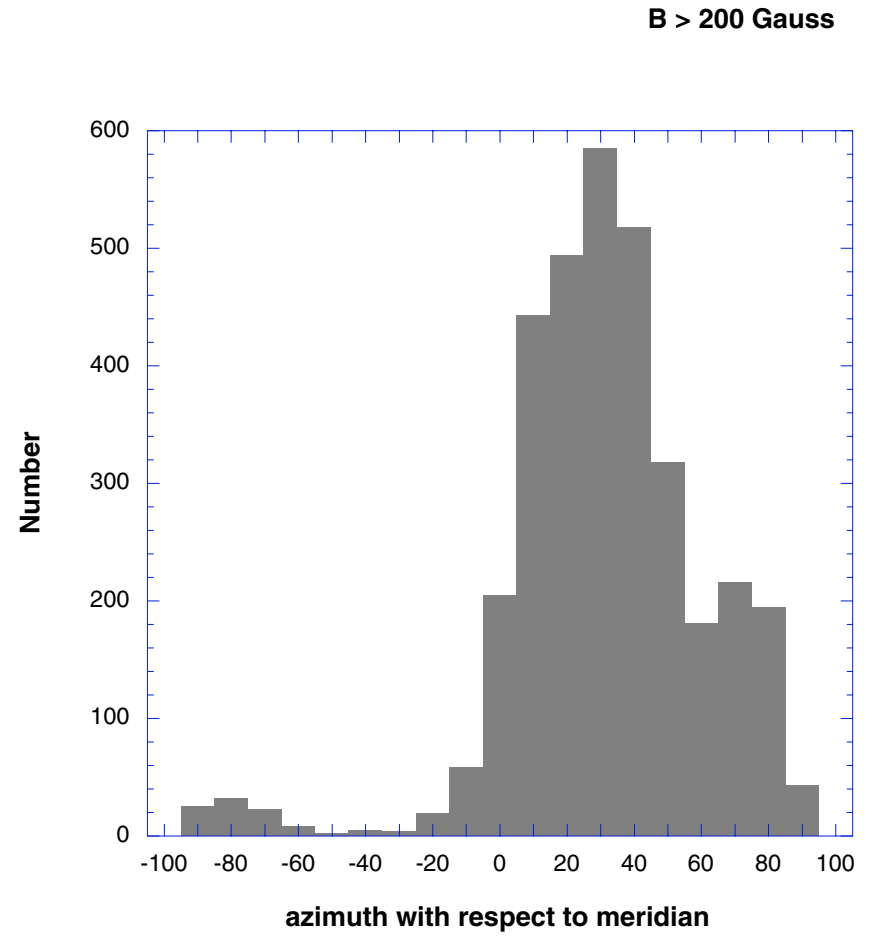

Fig. 6. Histogram of the azimuth direction in the central third of the map (in the filament zone). The azimuth is referred to the slit direction, which is also the meridian (map $O y$ axis). Only the points where the field strength $B_{\text {tot }}$ is larger than 200 Gauss were kept to build the histogram, which shows the homogeneity of the azimuth direction in the filament zone.

(slightly lower); $6302 \AA$ spectral resolution $31 \mathrm{~m} \AA$ for ASP, $24 \mathrm{~m} \AA$ for THEMIS (slightly better). The main difference lies in the instrumental polarization, which has however been calibrated in ASP (Skumanich et al. 1997), whereas THEMIS is a polarization-free telescope, and also on the multiline facility of THEMIS that is unique. Besides, the scientific goals of the ASP concern sunspots and active regions (Elmore et al. 2005), and no ASP observations of the magnetic field below a filament has been found.

\subsection{Effect of the filling factor}

The effect of an eventual unresolved filling factor for the magnetic field has however to be discussed, along with unresolved inhomogeneity of the atmosphere, which is not taken into account in the present magnetic derivation from polarization measurements. As shown by Landi Degl'Innocenti (1992b, Sect. 9; see also Landi Degl'Innocenti \& Landolfi 2004, Sect. 11.5), such a filling factor does not act in the same manner on the longitudinal and transverse fields. The longitudinal field strength scales as $1 / f$, where $f$ is the filling factor, whereas the transverse magnetic field strength scales as $1 / \sqrt{f}$. Thus, the magnetic field inclination also depends on the unknown $f$. However, assuming any given $f$ will not change the main conclusions of this work, namely: a) the field is inclined and b) its inclination and azimuth (i.e., the field direction) are quite homogeneous, except in the sunspot zone. Assuming $f<1$ changes differently the axis scales in Fig. 4, but does not change

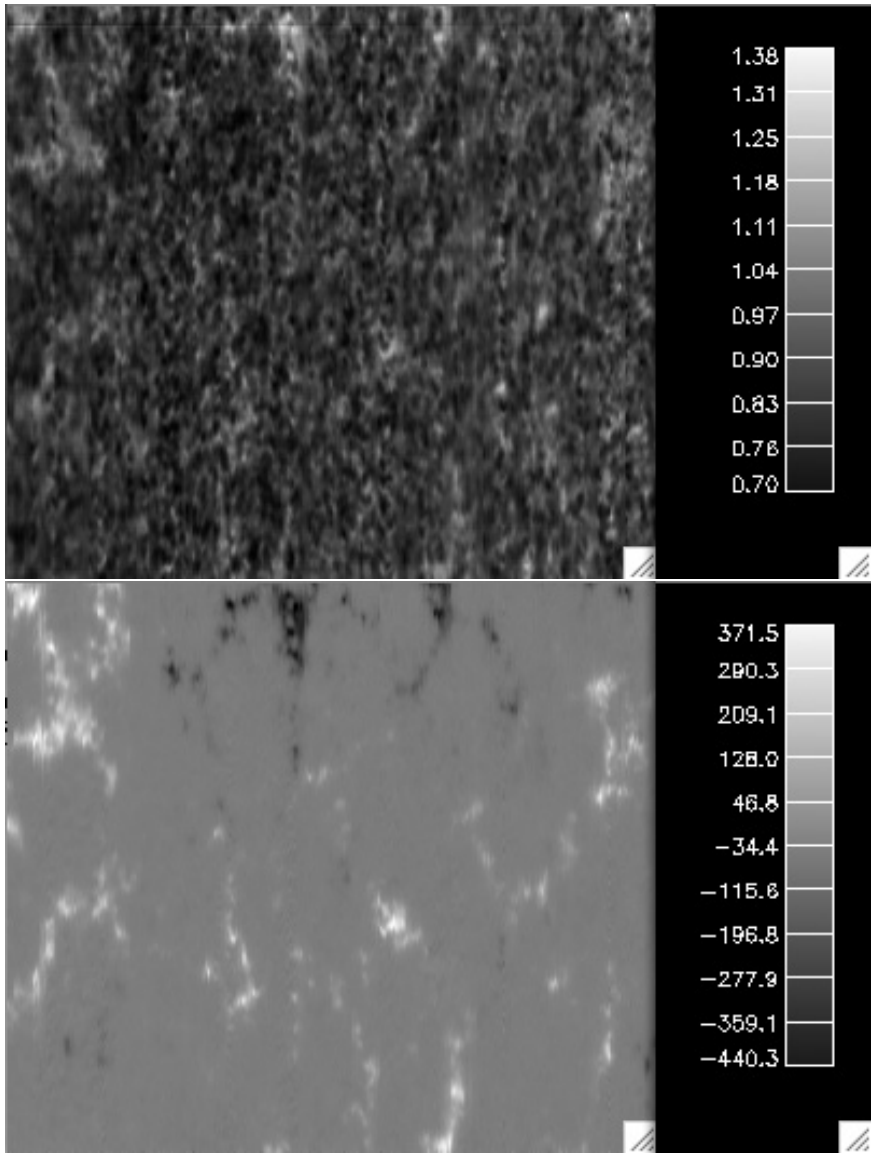

Fig. 7. For comparison, map of the $5576 \AA$ line center second derivative (line insensitive to the magnetic field) (top) and of the longitudinal magnetic field (bottom), in the central third of the full map (not corrected for anamorphosis). The second derivative at line center is given in units of the the average flat field profile one. Each structure of the longitudinal magnetic field is associated with a structure of larger second derivative with the same shape.

the result, which is that the scatter of points does not cut the axes. Even if the inclination angle is $f$-dependent, the fact that it is homogeneous remains true. The same conclusion holds for the field azimuth.

\subsection{An example of coupling between magnetic and thermodynamic parameters}

As explained in Sect. 4.2, the second derivative of the intensity profile was taken at the center of the non-magnetic Fe I 5576.1 A line. The map of this second derivative, scaled to the one of the average flat field profile, is displayed in the upper part of Fig. 7. The lower part of the figure shows the longitudinal magnetic field map, so that the correlation between the zones of longitudinal field and the zones of higher second derivative appears clearly. As the second derivative value was derived by applying the GAUSSFIT procedure (fitting by a Gaussian plus polynomial function having 6 free parameters), the second derivative at line center is related to the inverse of the line half-width. Thus, larger second derivatives (up to $40 \%$ larger) indicate smaller half-widths (of up 
to $20 \%$ smaller) in the magnetic regions. As line width is related to the line formation parameters, this indicates coupling between the magnetic and thermodynamic parameters of the atmosphere. If it is moreover assumed that there is a filling factor $f<1$ for the magnetic field in the magnetic regions, and if it is in addition assumed that the thermodynamic parameters of the non-magnetic part of the atmosphere are the same in and out the magnetic regions, such a high $20 \%$ variation factor is not compatible with a very small filling factor. Alternatively, the thermodynamic parameters have to be different inside and out of the magnetic regions, in particular for the non-magnetic part of the atmosphere.

\section{Conclusion}

By plotting a $240 \times 340$ arcsec map with a pixel size of 0.45 arcsec, we observed the following characteristics of the field vector: a) the field vector is neither vertical nor horizontal, but inclined; b) the field vector inclination and azimuth are quite homogeneous except in the sunspot zone. The method for interpreting the polarization in terms of magnetic field vector (taken from Rayrole 1967; and Semel 1967, for the longitudinal field; and from Landi Degl'Innocenti 1992a, for the transverse field, completed by a "calibration" procedure described in this paper), was tested on eight scattered points by comparing the results with those of the UNNOFIT inversion code (Landolfi et al. 1984), and the discrepancy does not exceed $14 \%$ on average. The atmosphere is assumed to be homogeneous, i.e. no filling factor is assumed for the magnetic field, but introducing a filling factor $f<1$ would not change the results a) and b) above.

Our map is centered on a filament that should lie along a neutral line. Parasitic polarities appear on both sides of the filament. Exact superimposition of the magnetic field map derived from the Fe I $6302.5 \AA$ line and of the $\mathrm{H} \alpha$ map, - which enabled the association of the parasitic polarities with the filament feet - has been possible because these two maps were obtained simultaneously, a unique facility available in the multiline mode of THEMIS.

The fundamental ambiguity is not solved, so that the orientation of the field on its direction is indeterminate. If it is assumed that the filament field is inversely oriented with respect to the neighboring photospheric field, as found in most quiescent filaments (Leroy et al. 1984; Bommier et al. 1994), then its chirality would be dextral, even though it is located in the southern hemisphere where the quiescent filament chirality should be sinistral. These incompatible features, probably a result of the non-quiescent type of this filament located in the vicinity of an active region, have to be further investigated by magnetic extrapolation of the filament field.

\section{References}

Arnaud, J., Mein, P., \& Rayrole, J. 1998, Proceedings of A Crossroads for European Solar \& Heliospheric Physics, Tenerife, 1998 March 23-27, ESA SP-417, 213

Auer, L. H., Heasley, J. N., \& House, L. L. 1977, Sol. Phys., 55, 47

Aulanier, G., \& Démoulin, P. 1998, A\&A, 329, 1125

Aulanier, G., \& Démoulin, P. 2003, A\&A, 402, 769

Bommier, V., \& Rayrole, J. 2002, A\&A, 381, 227

Bommier, V., \& Molodij, G. 2002, A\&A, 381, 241

Bommier, V., Landi Degl'Innocenti, E., Leroy, J. L., \& Sahal-Bréchot, S. 1994, Sol. Phys., 154, 231

Donati, J. F., Semel, M., Rees, D. E., Taylor, K., \& Robinson, D. 1990, A\&A, 232, L1

Elmore, D. F., Lites, B. W., Tomczyk, S., et al. 2005, Advanced Stokes Polarimeter, in NSO/SP: DST User's Manual, http://nsosp.nso.edu/dst/userman/instruments/ advanced-stokes-polarimeter/asp.html

Hagyard, M. J., Cumings, N. P., West, E. A., \& Smith, J. E. 1982, Sol. Phys., 80, 33

Harvey, J., Livingston, W., \& Slaughter, C. 1972, in Line Formation in the Presence of Magnetic Fields, High Altitude Observatory, NCAR (Colorado: Boulder), 227

Keller, C. U., \& NSO staff 1998, in Cool Stars, Stellar System and the Sun, ed. R. A. Donahue, \& J. A. Bookbinder, ASP Conf. Ser., 154, CD-636

Landi Degl'Innocenti, E. 1992a, Workshop Méthodes de détermination des champs magnétiques solaires et stellaires, Paris France, 15-16 janvier, ed. M. Faurobert-Scholl, H. Frisch, \& N. Mein, 7

Landi Degl'Innocenti, E. 1992b, in Solar Observations: Techniques and Interpretation, ed. F. Sanchez, M. Collados, \& M. Vazquez (Cambridge University Press), 73

Landi Degl'Innocenti, E., \& Bommier, V. 1993, ApJ, 411, L49

Landi Degl'Innocenti, E., \& Landolfi, M. 2004, Polarization in Spectral Lines (Kluwer Academ. Publ.)

Landolfi, M., \& Landi Degl'Innocenti, E. 1982, Sol. Phys., 78, 355

Landolfi, M., Landi Degl'Innocenti, E., \& Arena, P. 1984, Sol. Phys., 93, 269

Leroy, J. L., Bommier, V., \& Sahal-Bréchot, S. 1984, A\&A, 131, 33

Mickey, D. L., Canfield, R. C., LaBonte, B. J., et al. 1996, Sol. Phys., 168,229

Rayrole, J. 1967, Ann. Astrophys., 30, 257

Semel, M. 1967, Ann. Astrophys., 50, 513

Skumanich, A., Lites, B. W., Martínez Pillet, V., \& Seagraves, P. 1997, ApJS, 110, 357

Trujillo Bueno, J., Collados, M., Paletou, F., \& Molodij, G. 2001, in Advanced Solar Polarimetry, Proc. 20th NSO/Sac Peak Summer Workshop, ed. M. Sigwarth, ASP Conf. Ser., 236, 141 